\title{
Prevención precoz de hipertrofia ventricular izquierda en la hipertensión experimental y concentraciones de angiotensina II
}

\author{
Anita Gálvez ${ }^{a}$, María P. Ocaranza ${ }^{a, b}$, Sergio Lavandero ${ }^{a}$ y Jorge E. Jalil ${ }^{b}$ \\ aDepartamento de Bioquímica y Biología Molecular. Facultad de Química y Farmacia. Universidad de Chile. \\ Santiago. Chile. ${ }^{b}$ Departamento de Enfermedades Cardiovasculares. Facultad de Medicina. P. Universidad \\ Católica de Chile. Santiago. Chile.
}

Introducción. Las concentraciones de angiotensina II pueden inhibirse parcialmente durante la administración crónica de inhibidores de la enzima conversiva de la angiotensina (ECA), limitando desde el punto de vista clínico su eficacia en el tratamiento de la hipertensión arterial. Existen pocos estudios que relacionan directamente la actividad de la ECA y la prevención precoz de hipertrofia ventricular izquierda (HVI) secundaria a hipertensión arterial durante la administración de un inhibidor de la ECA (IECA).

Objetivo. Evaluar los efectos de la inhibición precoz de la ECA con perindopril sobre el desarrollo de hipertensión, HVI y concentraciones de angiotensina II plasmática y en el ventrículo izquierdo en el modelo Goldblatt en la rata (Gb; 2 riñones-un pinzado) a las 2 semanas de la cirugía.

Resultados. La presión arterial sistólica y la masa ventricular izquierda relativa aumentaron un 42 y un $20 \%$, respectivamente, en el grupo $\mathrm{Gb}(\mathrm{p}<0,001)$. Las actividades de ECA circulante y en el ventrículo izquierdo fueron significativamente mayores en las ratas $\mathrm{Gb}$ comparadas con los controles. Las concentraciones de angiotensina II plasmática y en el ventrículo izquierdo también aumentaron un 129 y un $800 \%$, respectivamente. El perindopril previno la aparición de hipertensión y el desarrollo de HVI, ya que inhibió la ECA plasmática (y en el ventrículo izquiedo), además de la angiotensina II circulante y en el ventrículo izquierdo.

Conclusiones. En este modelo experimental de HVI hipertensiva existe una activación temprana de la ECA plasmática y cardíaca. La administración precoz de un IECA previene el desarrollo de hipertensión e HVI al inhibir el aumento de angiontensina II en el plasma y el ventrículo izquierdo.

Palabras clave: Angiotensina. Enzima conversiva de la angiotensina. Hipertrofia. Perindopril.

\section{Early Prevention of Experimental Left Ventricular Hypertrophy in Experimental Hypertension and Angiotensin II Levels}

Introduction. Angiotensin II levels can be partially inhibited during chronic administration of angiotensin converting enzyme (ACE) inhibitors, limiting from a clinical point of view its efficacy in the treatment of hypertension. There are few studies relating ACE activity directly with early prevention of left ventricular hypertrophy (LVH) in systemic hypertension during the administration of an ACE inhibitor (ACEI).

Aim. To evaluate the effects of early ACE inhibition with perindopril on the development of hypertension, LVH and levels of angiotensin II (Ang II) in plasma as well as in LV in the rat Goldblatt model (Gb; 2 kidneys-1 clip), 2 weeks after surgery.

Results. Systolic blood pressure and relative LV mass increased by $42 \%$ and $20 \%$ respectively, in the Gb group $(p<0.001)$. Plasma and LV ACE activities were significantly higher in the $\mathrm{Gb}$ rats compared with the control rats. Plasma and LV Ang II levels also increased by $129 \%$ and $800 \%$, respectively. Perindorpil prevented hypertension and LVH development by inhibiting plasma ACE (and also LV ACE), and also circulation Ang II in plasma and in the LV.

Conclusions. In this experimental model of hypertensive $L V H$, there is an early activation of plasma and cardiac ACE. Early administration of an ACE inhibitor prevents the development of hypertension and LVH by inhibiting the increases of plasma and LV Ang II.

Key words: Angiotensin. Angiotensin converting enzyme. Hypertrophy. Perindopril.
Financiado parcialmente por Fondecyt Proyectos 1961065 y 2970021 , por un Programa de Intercambio ECOS-Conicyt y por una beca internacional de la Uchida Foundation.

Correspondencia: Dr. J.E. Jalil.

Pontificia Universidad Católica de Chile. Facultad de Medicina.

Departamento de Enfermedades Cardiovasculares.

Marcoleta, 367. Santiago de Chile.

\section{INTRODUCCIÓN}

La hipertrofia ventricular izquierda (HVI), usualmente causada por hipertensión arterial, aumenta de manera considerable el riesgo de infarto del miocardio, muerte súbita, accidentes cerebrovasculares e in- 


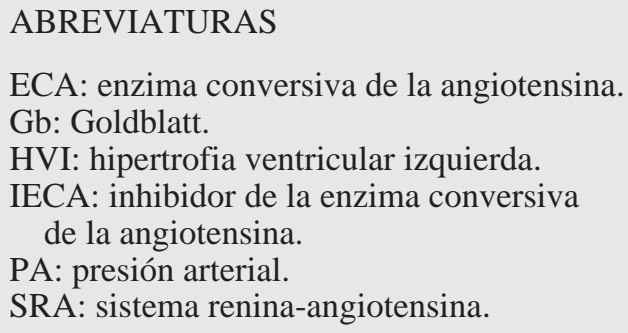

suficiencia cardíaca ${ }^{1}$. Modificaciones en las concentraciones de angiotensina II, catecolaminas, en algunos factores de crecimiento celular (p. ej., el factor de crecimiento análogo de insulina), endotelina y en la presión arterial (PA) contribuyen directamente al desarrollo de HVI patológica. Es posible que el desarrollo y las características moleculares de la HVI estén modulados mayoritariamente por el sistema renina-angiotensina (SRA) que por la PA propiamente.

El bloqueo farmacológico del SRA en ratas con pinzamiento (banding) de la aorta ascendente (donde la sobrecarga de presión se mantiene pero el SRA es bloqueado) disminuye en mayor grado el desarrollo de HVI que el tratamiento con el fármaco antihipertensivo hidralazina (el cual normaliza la presión arterial pero no inhibe al SRA) ${ }^{2}$.

Tanto la actividad de la enzima conversiva de la angiotensina I (ECA, dipeptidil carboxipeptidasa I, cininasa II; EC 3.4.15.1) y su ARN mensajero están incrementados en el ventrículo hipertrófico, y el aumento de la velocidad de producción de la angiotensina II se asocia con alteraciones en las propiedades diastólicas del corazón hipertrofiado ${ }^{3}$. En la hipertensión arterial, mientras el corazón desarrolla HVI patológica, podría ocurrir que la actividad de ECA circulante y/o local (tisular) aumentara. La angiotensina II y sus metabolitos son detectados en el plasma durante la administración crónica de inhibidores de ECA, lo que indica que la inhibición del SRA no es completa; esto puede limitar la eficiencia clínica de estos inhibidores en el tratamiento de la hipertensión arterial y daño cardíaco ${ }^{4}$. Existen pocos datos sobre la relación entre la actividad de ECA y la prevención precoz de la HVI hipertensiva con el uso de inhibidores de la ECA (IECA). Una mejor comprensión de esta relación ayudaría a evaluar las ventajas terapéuticas de la inhibición exlusiva de la ECA o del bloqueo del SRA en otro nivel (p. ej., utilizando bloquedores del receptor tipo 1 de la angiotensina II o bloquedores del receptor de aldosterona) en la prevención y tratamiento de esta condición patológica. No existen estudios del efecto de los IECA en la prevención precoz de la HVI inducida por sobrecarga de presión ni tampoco una evaluación simultánea de las concentraciones de angiotensina II. Hemos evaluado aquí el efecto del IECA perindopril -administrado de forma precoz y a dosis conocidamente antihipertensivas y capaces de inhibir la ECA-sobre los valores de presión arterial, HVI, actividad de la ECA circulante y también sobre las concentraciones de angiotensina II en el plasma y el ventrículo izquierdo en el modelo Goldblatt en la rata (2 riñones-un pinzado, $2 \mathrm{r}-1 \mathrm{p}$ ) a las 2 semanas de la intervención quirúrgica.

\section{MATERIAL Y MÉTODO}

\section{Modelo experimental de hipertrofia ventricular izquierda hipertensiva}

Para los experimentos se siguieron las recomendaciones de la Guía para el Cuidado y Uso de Animales de Laboratorio (publicación NIH $\mathrm{N}^{\mathrm{o}}$ 85-23, revisada en 1985) y aprobada por la Comisión de Investigación de la P. Universidad Católica de Chile. La hipertensión experimental y la HVI fueron inducidas en la rata usando el modelo Goldblatt (Gb; 2r-1p) como se ha descrito con anterioridad ${ }^{5}$. Brevemente, bajo anestesia con éter, ratas macho Sprague-Dawley (80-100 g) fueron sometidas a una pequeña incisión abdominal tras la cual se colocó una pinza de plata $(0,2 \mathrm{~mm}$ de espesor interno) alderedor de la arteria renal izquierda, dejando la arteria renal derecha intacta. Todas las ratas sobrevivieron a la operación e ingresaron en el estudio. Los animales fueron mantenidos bajo condiciones controladas de luz y oscuridad y tuvieron libre acceso a agua y comida. Las ratas del grupo control fueron sometidas a la misma incisión abdominal pero sin la colocación de la pinza alrededor de la arteria renal izquierda.

La presión arterial sistólica (PAS) se midió con método pletismográfico en la cola (tail cuff) $24 \mathrm{~h}$ antes del sacrificio. Las ratas fueron pesadas y decapitadas. Los corazones fueron rápidamente extraídos. La aorta, la arteria pulmonar y los ventrículos fueron separados con cuidado y lavados extensamente con solución salina fría para limpiar toda la sangre. El grado de HVI se cuantificó usando la relación entre el peso del ventrículo izquierdo y la masa corporal (masa ventricular izquierda relativa [MRVI VI] * 100/peso corporal).

\section{Grupos experimentales}

Se usaron 3 grupos experimentales: Gb, control y Gb-Per (11 animales por grupo). El IECA perindopril (Per) se administró en dosis de $4 \mathrm{mg} / \mathrm{kg} /$ día por gavaje diario, comenzando inmediatamente después de la cirugía y continuando durante 2 semanas. El día del sacrificio se recolectó sangre para medir la actividad de la ECA plasmática y las concentraciones de angiotensina II. Los ventrículos izquierdos se lavaron exhaustivamente en una solución salina, se congelaron en nitrógeno líquido y se almacenaron a $-80{ }^{\circ} \mathrm{C}$ para la determinación de la angiotensina II tisular. 


\section{Determinación de la actividad de la ECA en el plasma y el ventrículo izquierdo}

La acividad de la ECA se midió por fluorimetría por medio de la hidrólisis de Z-fenil-L-histidil-L-leucina (Bachem Bioscience Inc., EE.UU.), un sustrato análogo de la $\mathrm{ECA}^{6-8}$. El producto L-histidil-L-leucina se cuantificó por espectrofluorimetría, para lo cual se usó un espectrofotómetro luminiscente (Perkin Elmer LS 50b, EE.UU.). Las determinaciones se realizaron por duplicado. La actividad de la ECA plasmática se expresó en $\mathrm{U} / \mathrm{ml}$ ( $1 \mathrm{U}=1 \mathrm{nmol} \mathrm{L}$-histidil-L-leucina/min). Para la medición de la actividad de la ECA tisular, 200 $\mathrm{mg}$ del ventrículo izquierdo se homogeneizaron con tampón tris-HCl, 50 mmol/l; $\mathrm{pH} \mathrm{8,0;} \mathrm{NaCl} 1 \%$. El extracto se centrifugó a $1.000 \mathrm{~g}$ por $15 \mathrm{~min}$ a $4{ }^{\circ} \mathrm{C}$ y el sobrenadante resultante se recentrifugó a $105.000 \mathrm{~g}$ por $30 \mathrm{~min}$ a $4{ }^{\circ} \mathrm{C}$. La pella se resuspendió en tampón CHAPS $8 \mathrm{mmol} / \mathrm{l}$. La concentración de proteínas se midió por Bradford usando seroalbúmina de bovino como estándar9. La actividad de ECA tisular se expresó en $\mathrm{U} / \mathrm{mg}$ de proteína.

\section{Recolección de muestras para la medición de la angiotensina II plasmática}

Las muestras de sangre para la medición de la angiotensina II se recogieron en tubos de poliestireno preenfriados, como describen Admiraal et $\mathrm{al}^{10}$, fueron inmediatamente transferidas a tubos de poliestireno y centrifugadas a $3.000 \mathrm{~g}$ por $10 \mathrm{~min}$ a $4{ }^{\circ} \mathrm{C}$. Las muestras de plasma se almacenaron a $-80^{\circ} \mathrm{C}$ y se determinaron dentro de la semana siguiente. Los plasmas fueron directamente aplicados en cartridges Sep-pack, como se describe a continuación.

\section{Recolección, extracción y determinación de la angiotensina II en el ventrículo izquierdo}

Los corazones se extrajeron rápidamente y las aurículas y los ventrículos derechos e izquierdos fueron separados. Estos tejidos se congelaron inmediatamente en nitrógeno líquido y se guardaron a $-80{ }^{\circ} \mathrm{C}$ hasta su determinación. Los ventrículos izquierdos se homogeneizaron por Ultraturrax en solución de extracción fría. Los homogeneizados se centrifugaron a $4{ }^{\circ} \mathrm{C}$ y los sobrenadantes se concentraron por evaporación. El remanente de cada sobrenadante se diluyó en $0,1 \% \mathrm{H}_{3} \mathrm{PO}_{4} \mathrm{y}$ se recentrifugó a $4{ }^{\circ} \mathrm{C}$. El sobrenadante se diluyó con $0,02 \% \mathrm{H}_{3} \mathrm{PO}_{4}$ y se concentró por adsorción reversible en cartridges de octadecil silil sílica (SepPak C-18, Waters, Milford, MA). Los cartridges se activaron con metanol y se equilibraron con 2 lavados de agua destilada fría. Las muestras se aplicaron a los cartridges a 4 ${ }^{\circ} \mathrm{C}$, seguido de 2 lavados con agua destilada fría y con hexano (sólo para las muestras de tejido). Las angiotensinas adsortas se eluyeron con metanol al $90 \% \mathrm{y}$, posteriormente, el metanol se evaporó a $4{ }^{\circ} \mathrm{C}$, usando un concentrador Speed-Vac. Las angiotensinas se separaron por HPLC de fase reversa con el método de Nussberger et $\mathrm{al}^{11}$, usando una columna Bondapak C18. El extracto concentrado del SepPak se disolvió antes de la inyección. Los eluidos se recogieron en tubos de poliestireno en fracciones que contenían BSA al $0,1 \%$. Las fracciones que contenían angiotensina II se neutralizaron con $0,5 \mathrm{~mol} / \mathrm{l}$ de $\mathrm{NaOH}$. Las concentraciones de angiotensina II se midieron por radioinmunoanálisis $^{12}$. La recuperación de la angiotensina II después de la homogeneización, extracción y separación por HPLC se determinó agregando [ $\left.{ }^{125} \mathrm{I}\right]$ angiotensina II al tejido $(10.000 \mathrm{cpm} / \mathrm{g})$ antes de la homogeneización.

\section{Análisis estadístico}

Los resultados se expresan como media \pm error estándar. Para las comparaciones múltiples se usó el análisis de la variancia con un factor (ANOVA) y posteriormente la prueba de Student-Newman-Keuls. También se utilizó el test de correlación lineal. Un valor de $\mathrm{p} \leq 0,05$ fue considerado como estadísticamente significativo.

\section{RESULTADOS}

\section{Estudios hemodinámicos e hipertrofia ventricular izquierda}

Como se expone en la tabla 1 , se observó hipertensión arterial a las 2 semanas en el grupo $\mathrm{Gb}(\mathrm{F}=54$; $\mathrm{p}$ $<0,001)$ comparado con el grupo control. También se observó un grado significativo de HVI (20\% de aumento en MRVI comparado con las ratas controles) en el grupo $\mathrm{Gb}(\mathrm{F}=11,6 ; \mathrm{p}<0,001)$. Ambos efectos fueron totalmente prevenidos por la administración de perindopril (tabla 1). Se observaron pequeñas diferencias

TABLA 1. Efecto de un inhibidor de la enzima conversiva de angiotensina I (perindopril) administrado precozmente sobre el desarrollo de hipertrofia ventricular izquierda hipertensiva

\begin{tabular}{llccccc}
\hline & Controles & Gb & Gb-Per & & \\
\cline { 2 - 4 } $\mathbf{N}$ & $\mathbf{1 1}$ & $\mathbf{1 1}$ & $\mathbf{1 1}$ & $\mathbf{F}$ & $\mathbf{P}$ \\
\hline PAS (mmHg) & $103 \pm 4$ & $146 \pm 4^{*}$ & $96 \pm 3$ & 54 & $<0,001$ \\
Peso corporal (g) & $199 \pm 4$ & $234 \pm 7^{\star}$ & $182 \pm 7$ & 18,1 & $<0,001$ \\
Masa VI (mg) & $540 \pm 18$ & $760 \pm 57^{*}$ & $440 \pm 18$ & 20,1 & $<0,001$ \\
MRVI (mg*100/g) & $272 \pm 6$ & $323 \pm 17^{*}$ & $242 \pm 10$ & 11,1 & $<0,001$ \\
Masa VD (mg) & $150 \pm 6$ & $160 \pm 12$ & $110 \pm 9^{* *}$ & 9,1 & $<0,001$ \\
\hline
\end{tabular}

${ }^{*} p<0,05$ frente a controles y Gb-Per (Student-Newman-Keuks test post-ANOVA), " $p<0,05$ frente a controles-2s y Gb (Student-Newman-Keuks test postANOVA). Los valores se expresan como media \pm error estándar. Gb: ratas Goldblatt, a las 2 semanas de operadas; Per: perindopril; PAS: presión arterial sistólica; VI: ventrículo izquierdo; VD: ventrículo derecho; MRVI: masa relativa del ventrículo izquierdo. 


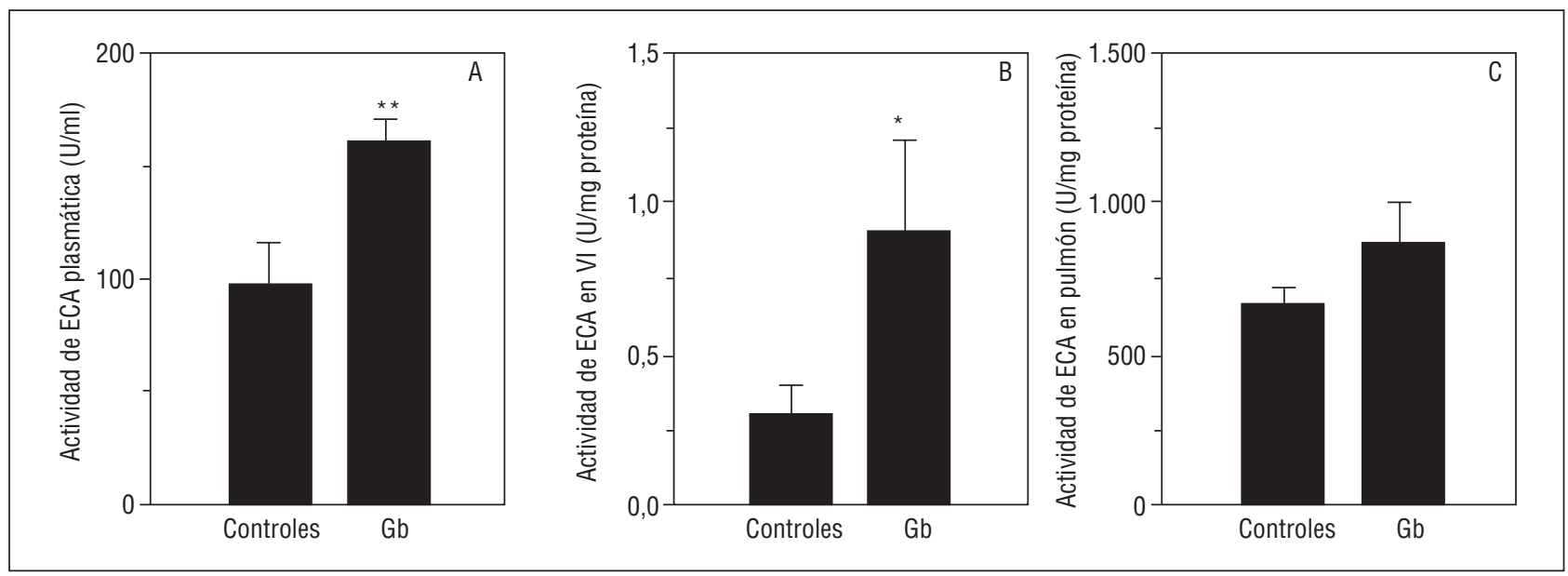

Fig. 1. Actividad de la enzima conversiva de la angiotensina (ECA) en plasma (A), ventrículo izquierdo (B) y pulmón (C) durante el desarrollo precoz de hipertrofia ventricular izquierda (HVI) hipertensiva. La actividad de ECA está expresada en U/mI de plasma $0 \mathrm{U} / \mathrm{mg}$ de proteína tisular. $1 \mathrm{U}$ : 1 nmol L-histidil-L-leucina/min. Controles $(n=11)$ y Goldblatt $(G b ; n=11)$. Valores expresados como media \pm error estándar. ${ }^{*} p<0,050{ }^{* *} p<0,01$ frente a grupo control (post-ANOVA).

en la masa corporal en los animales controles comparados con las ratas Gb. Se observó una correlación significativa entre la PAS y la MRVI $(\mathrm{r}=0,51 ; \mathrm{p}<0,01)$.

\section{Actividad de la ECA plasmática y tisular}

Las actividades de ECA en plasma, pulmón y ventrículo izquierdo a las 2 semanas de la intervención quirúrgica se exponen en la figura 1 . La actividad de la ECA plasmática fue un $60 \%$ mayor en las ratas $\mathrm{Gb}$ comparada con sus respectivos controles (fig. 1A) y en el ventrículo izquierdo fue 3 veces mayor en el grupo Gb que en los animales del grupo control ( $p<0,001)$ (fig. 1B). No hubo diferencias en las actividades de la ECA pulmonar entre los grupos Gb y control (fig. 1C).

No se encontró correlación entre la PAS y la actividad de la ECA en el plasma o el ventrículo izquierdo. La MRVI no se correlacionó con la actividad de la ECA plasmática.

\section{Concentraciones de angiotensina II en el plasma y el ventrículo izquierdo}

En experimentos preliminares con 2 inhibidores diferentes de la ECA no encontramos inhibición de la actividad de la ECA en el ventrículo izquierdo, mientras que simultáneamente observamos una reducción del $99 \%$ en la actividad de la ECA plasmática (datos no expuestos). Por esta razón, hemos medido las concentraciones de angiotensina II en el plasma y el ventrículo izquierdo después de 2 semanas de tratamiento con perindopril ( $4 \mathrm{mg} / \mathrm{kg} /$ día, vía gavaje) para estimar así el grado de inhibición de la ECA en el ventrículo izquierdo. Campbell et al encontraron que la dosis de perindopril utilizada en el presente estudio inhibe casi completamente la actividad de la ECA plasmática ${ }^{12}$, lo cual hemos confirmado en experimentos preliminares.

Las concentraciones de angiotensina II en el plasma y el ventrículo izquierdo (fig. 2) aumentaron de manera significativa en las ratas $\mathrm{Gb}$ a las 2 semanas de la intervención quirúrgica (129 y 800\%, respectivamente; $\mathrm{F}=12,2 ; \mathrm{p}<0,01$ y 5,$9 ; \mathrm{p}<0,01)$ y también se corre-

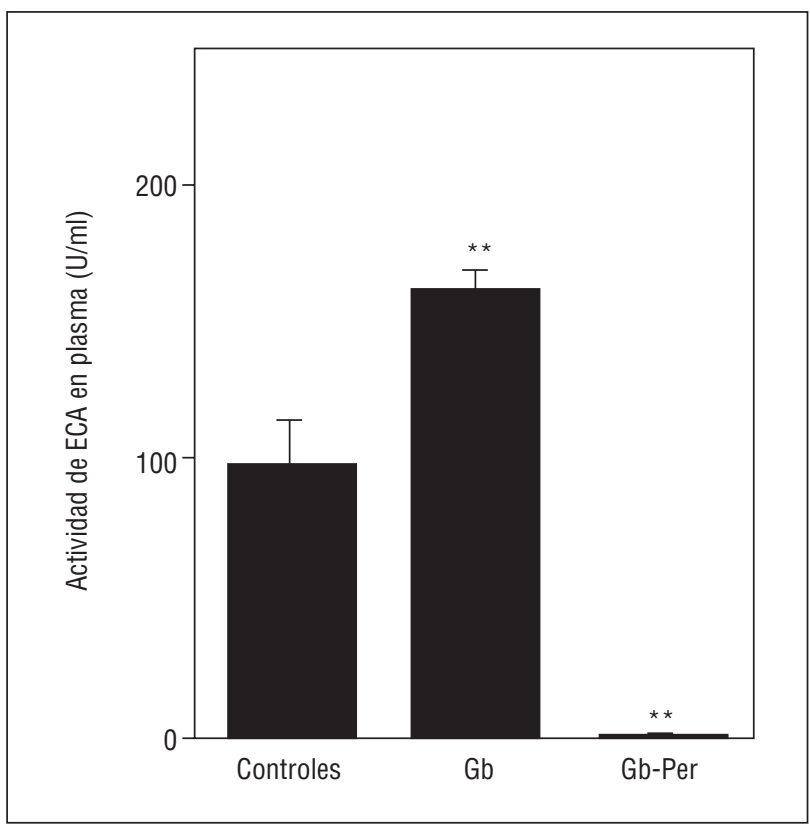

Fig. 2. Efecto de la administración precoz del inhibidor de la enzima conversiva de la angiotensina (ECA) perindopril sobre la actividad de la ECA plasmática durante la prevención precoz de hipertrofia ventricular izquierda (HVI) hipertensiva. Se determinó la actividad de la ECA plasmática $(\mathrm{U} / \mathrm{ml})$ en los grupos control $(n=11)$, en las ratas Goldblatt a las 2 semanas de la intervención $(\mathrm{Gb}, \mathrm{n}=11)$ y en las ratas tratadas con perindopril (4 mg/kg/día, administrado por gavaje diario) (Gb-Per, $n=11$ ). Valores expresados como media \pm error estándar. ${ }^{* \star} p<0,05$ frente a grupo control (post-ANOVA). Gb: Goldblatt, Per: perindopril. 
Fig. 3. Efecto de la administración precoz del IECA perindopril sobre las concentraciones de angiotensina II en el plasma y el ventrículo izquierdo durante la prevención precoz de la hipertrofia ventricular izquierda (HVI) hipertensiva. Las concentraciones de angiotensina II en el plasma y el ventrículo izquierdo ( $\mathrm{pg} / \mathrm{ml}$ y $\mathrm{pg} / \mathrm{mg}$ tejido, respectivamente) se midieron después de la administración de perindopril (4 $\mathrm{mg} / \mathrm{kg} / \mathrm{día}$, administrado por gavaje diario), que se inició inmediatamente después de la cirugía y se continuó durante 2 semanas (Gb-Per). Las concentraciones de angiotensina II en el plasma $(A)$ y en el ventrículo izquierdo (B) se midieron por HPLC y radioinmunoanálisis (véase el apartado Material y métodos). Valores expresados como media \pm error estándar. ${ }^{* *} p<0,05$ frente a grupo control (post-ANOVA). Gb: Goldblatt; Per: perindopril. lacionaron significativamente entre sí $(\mathrm{r}=0,6 ; \mathrm{p}<$ 0,002). La actividad de la ECA circulante se correlacionó de manera significativa con las concentraciones plasmáticas de angiotensina II ( $\mathrm{r}=0,51 ; \mathrm{p}<0,02)$.

La administración preventiva de perindopril durante 2 semanas comenzando inmediatamente después de la cirugía inhibió completamente la actividad de la ECA circulante (fig. 3), causando una gran disminución de las concentraciones de angiotensina II en el plasma y en el ventrículo izquierdo (fig. 2). Las concentraciones de angiotensina II plasmática se correlacionaron significativamente con la PAS $(\mathrm{r}=0,52 ; \mathrm{p}<0,005)$ y con la MRVI $(r=0,48 ; p<0,005)$. Los valores de angiotensina II en el ventrículo izquierdo se correlacionaron con la PAS $(r=0,5 ; p<0,01)$ y con la masa ventricular izquierda $(\mathrm{r}=0,41 ; \mathrm{p}<0,03)$.

\section{DISCUSIÓN}

Estos resultados ponen de manifiesto que: a) la actividad de la ECA y las concentraciones de angiotensina II en el plasma y el ventrículo izquierdo aumentan precozmente en la HVI hipertensiva en este modelo experimental, y $b$ ) el uso preventivo de un inhibidor de la ECA (en este caso perindopril) normalizó la actividad de la ECA y las concentraciones de angiotensina II en el plasma y en el ventrículo izquierdo, y además previno el desarrollo de HVI, lo que se asoció a una normalización de la presión arterial.

La ECA es una zinc metalopeptidasa unida a membrana que está localizada principalmente en el endotelio de los vasos, sobre todo en la circulación pulmonar ${ }^{13}$. También se encuentra en la superficie de algunas células epiteliales, en las células mononucleares circulantes, macrófagos, células germinales masculinas y en el plasma o suero ${ }^{13}$. La ECA circulante proviene proba- blemente de células vasculares endoteliales. En el plasma y/o en células endoteliales la ECA convierte el decapéptido inactivo angiotensina I en el octapéptido activo angiotensina $\mathrm{II}^{13}$. En humanos, las concentraciones de ECA en el plasma varían durante el crecimiento: son elevadas durante la infancia y luego disminuyen, para permanecer estables durante la etapa adulta ${ }^{14}$.

En la hipertensión arterial, el aumento de las concentraciones de angiotensina II induce fibrosis miocárdica e HVI patológica ${ }^{15-17}$. Incluso a concentraciones subhipertensivas, la angiotensina II induce daño y fibrosis miocárdica ${ }^{18}$. La angiotensina II es también un potente vasoconstrictor que estimula la síntesis de proteínas en el corazón aislado de rata ${ }^{19}$, la secreción de aldosterona y el crecimiento tanto del músculo liso como de miocitos cardíacos. La angiotensina II también estimula la síntesis de colágeno tipo I en células intersticiales en cultivo $^{20}$. Además de la angiotensina II existen otros factores que estimulan la fibrogénesis en el miocardio, como la aldosterona, la endotelina y el factor de transformación tisular beta. Por otro lado, existen factores que contrarrestan los factores mencionados previniendo el desarrollo de fibrosis en el miocardio, como la bradicinina, el óxido nítrico, el péptido natriurético auricular y los glucocorticoides ${ }^{21}$. Es posible que genotipos que determinen concentraciones más elevadas de factores estimuladores (o menores valores de factores inhibidores) de fibrosis miocárdica puedan producirla con mayor facilidad frente a un mismo estímulo fibrogénico.

Challah et al observaron un aumento de la actividad de la ECA plasmática después de 2 semanas de la cirugía (pero no después de 4 y 12 semanas) usando el mismo modelo experimental que nosotros ${ }^{22}$. En la HVI hipertensiva establecida (Gb 9 semanas) nosotros observamos previamente que hay una activación de la ECA en el ventrículo izquierdo pero no en el plasma 
(datos no expuestos). Un aumento en la actividad de la ECA plasmática indica un incremento en la forma secretada de la ECA (ECA soluble), pero el origen de este aumento -endotelial o de otras vías- aún no ha sido determinado completamente.

Un aumento de la actividad de la ECA en el ventrículo izquierdo y de la expresión de su correspondiente ARN mensajero también se ha encontrado después de 2 y 4 semanas de cirugía, pero no después de 12 semanas en el mismo modelo experimental ${ }^{22}$. Varios estudios han demostrado que la actividad de la ECA en el ventrículo izquierdo aumentó en diferentes modelos de hipertrofia patológica ${ }^{23-25}$. Por ejemplo, en ratas con infarto al miocardio experimental, un aumento de la actividad de la ECA en el septum interventricular se debe a la inducción de su ARN mensajero y a una mayor síntesis local ${ }^{26}$. En nuestro estudio, el uso de un inhibidor de la ECA (perindopril) por un corto período de tiempo previno el desarrollo de HVI, lo que se asoció a una disminución de la presión arterial y a una inhibición simultánea de la actividad de la ECA circulante y en el ventrículo izquierdo.

Uno de los aspectos morfológicos característicos de la HVI patológica es el aumento del colágeno perivascular e intersticial ${ }^{27,28}$ con disfunción diastólica temprana y sistólica tardía del ventrículo izquierdo ${ }^{29-33}$. En la HVI hipertensiva, la sobreproducción de ECA en el miocardio parece indicar un remodelamiento intersticial, pero no hipertrofia de los cardiomiocitos ${ }^{22}$. Las células miocárdicas intersticiales responsables del aumento de la actividad de la ECA podrían ser activadas por fibroblastos, células inflamatorias, células musculares lisas activadas o todas ellas. Por tanto, en nuestro grupo $\mathrm{Gb}$ no tratado la activación por algunas de estas células podría ser la responsable del aumento de la actividad de la ECA en el ventrículo izquierdo.

La disminución de la actividad de la ECA en el ventrículo izquierdo durante la inhibición de la ECA ha sido observada previamente en ratas normales tratadas con quinapril ${ }^{34}$, en ratas con pinzamiento de la aorta abdominal $^{35} \mathrm{y}$ en ratas espontáneamente hipertensas ${ }^{36}$. También se ha observado usando el IECA ramipril en ratas transgénicas (TGR; mREN2) con hipertensión crónica $^{37}$ y, además, con enalapril y perindopril en ratas postinfarto de miocardio ${ }^{38,39}$.

Investigaciones anteriores han determinado las concentraciones de angiotensina II en el ventrículo izquierdo en ratas postinfarto de miocardio tratadas con perindopril ${ }^{40}$, también en ratas con fístula aortocava tratadas con quinapril ${ }^{41}$ pero no en la prevención precoz de HVI hipertensiva en el modelo Goldblatt. Esta observación tiene implicancias clínicas potenciales, ya que enfatiza la prevención (o el retardo de su desarrollo) de la HVI patológica en la hipertensión arterial dependiente de angiotensina II utilizando un IECA.

Resultan llamativos los cambios en la masa ventricular derecha con el tratamiento preventivo, ya que ésta disminuye significativamente con perindopril. En esta cavidad sólo deja de actuar como estímulo hipertrófico la angiotensina II, a diferencia de lo que sucede con la prevención de la HVI, en que en el ventrículo izquierdo dejan de actuar dos estímulos hipertrofiantes, como la sobrecarga hemodinámica y la angiotensina II. Al respecto, se ha sugerido que la hipertrofia miocitaria sería más dependiente de la sobrecarga hemodinámica, mientras que la angiotensina II y la aldosterona regularían la acumulación de colágeno en ambos ventrículos ${ }^{42}$.

En este estudio no hemos determinado directamente la actividad de ECA en el ventrículo izquierdo debido a que en experimentos piloto previos no pudimos detectar su inhibición, mientras que simultáneamente observamos $<1 \%$ de actividad de ECA en plasma (datos no expuestos). Esta subestimación de la inhibición de la ECA en el ventrículo izquierdo se podría explicar por el hecho de que el procedimiento puede tomar en nuestro caso $3 \mathrm{~h}$ a partir del momento de la remoción del tejido hasta el final de la determinación. Durante este período, los IECA unidos a la ECA tisular podrían disociarse y subestimarse así el grado verdadero de inhibición de ECA, como ha sido previamente comunicado por Urata et $\mathrm{al}^{43}$ y Kinoshita et $\mathrm{al}^{44}$. La medición de angiotensina II en el ventrículo izquierdo se ha considerado como una forma precisa para determinar la actividad de ECA al utilizar IECA en tejido cardía$\mathrm{co}^{12,43,45}$. También es posible que el perindopril, igual que otros IECA, adicionalmente pueda actuar inhibiendo la degradación de bradicinina ${ }^{12}$. Algunos estudios, sin embargo, han demostrado que la prevención de HVI secundaria a hipertensión vasculorrenal por IECA es independiente del sistema renina-angiotensina y de la presión arterial ${ }^{46,47}$.

\section{Limitaciones del estudio}

Hubiera sido interesante incluir un grupo de ratas hipertensas tratadas con una dosis no hipotensora de perindopril para dilucidar el mecanismo de prevención de la HVI. En el modelo utilizado descienden simultáneamente la presión arterial y las concentraciones circulantes y miocárdicas de angiotensina II tras la administración de perindopril, lo cual hace más difícil delimitar la contribución de cada uno de ambos mecanismos al efecto del fármaco sobre la HVI. Ésta es una limitación del estudio, que estuvo diseñado para evaluar el efecto de dosis conocidamente antihipertensivas y capaces de inhibir la ECA de perindopril sobre la prevención precoz de la HVI.

\section{Conclusiones}

En este modelo experimental de HVI hipertensiva dependiente de angiotensina II existe una activación precoz de la ECA circulante y en el ventrículo izquierdo. La administración precoz de un IECA en una dosis 
antihipertensiva inhibe la ECA circulante (y muy probablemente la ECA en el ventrículo izquierdo), normaliza las concentraciones elevadas de angiotensina II en el plasma y el ventrículo izquierdo y previene (pudiendo eventualmente regresar y retardar) la aparición de HVI.

\section{BIBLIOGRAFÍA}

1. Levy D, Garrison RJ, Savage DD, Kannel WB, Castelli WP. Prognostic implications of echocardiographically determined left ventricular mass in the Framingham Heart Study. N Engl J Med 1990; 322: 1561-1566.

2. Bruckschlegel G, Holmer SR, Jandeleit K, Grimm D, Muders F, Kromer EP et al. Blockade of the renin-angiotensin system in cardiac pressur-overload hypertrophy in rats. Hypertension 1995; 25: 250-259.

3. Schunkert H, Dzau V, Tang SS, Hirsch A, Apstein C, Lorell B. Increased rat cardiac angiotensin converting enzyme activity and mRNA expression in pressure overload left ventricular hypertrophy. J Clin Invest 1990; 86: 1913-1920.

4. Van Den Meiracker AH, Man in't veld AJ, Admiraal PJJ, Ritsema Van Eck HJ, Boomsma F, Derkx FHM et al. Partial escape of angiotensin converting enzyme (ACE) inhibition during prolonged ACE inhibitor treatment: does it exist and does it affect the antihypertensive response? J Hypertens 1992; 10: 803-812.

5. Dussaillant G, González H, Céspedes C, Jalil JE. Regression of left ventricular hypertrophy in experimental renovascular hypertension: diastolic dysfunction depends more on myocardial collagen than mass. J Hypertens 1996; 14: 1117-1123.

6. Friedland J, Silverstein E. A sensitive fluorimetric assay for serum angiotensin-converting enzyme. Am J Clin Pathol 1975; 66: 416-424.

7. Piquilloud Y, Reinharz JA, Roth M. Studies on the angiotensin converting enzyme with different substrates. Biochim Biophys Acta 1970; 206: 136-142.

8. Jalil JE, Ocaranza MP, Piddo AM, Jalil R. Reproducibility of plasma angiotensin-converting enzyme activity in human subjects determined by fluorimetry with Z-phenylalanine-histidyl-leucine as substrate. J Lab Clin Med 1999; 133: 501-506.

9. Bradford M. A rapid and sensitive method for the quantitation of microgram quantities of protein utilizing the principle of protein dye binding. Anal Biochem 1976; 72: 248-253.

10. Aadmiral PJJ, Derkx FHM, Danser AHJ, Pieterman H, Schalekamp MADH. Metabolism and production of angiotensin I in different vascular beds in subjects with hypertension. Hypertension 1990; 15: 44-55.

11. Nussberger J, Brunner DB, Waeber B, Brunner HR. Specific measurement of angiotensin metabolites and in vitro generated angiotensin II in plasma. Hypertension 1986; 8: 476-482.

12. Campbell DJ, Kladis A, Duncan AM. Effects of converting enzyme inhibitors on angiotensin and bradykinin peptides. Hypertension 1994; 23: 439-449.

13. Soubrier F, Hubert C, Testut P, Nadaud S, Alhenc-Gelas F, Corvol P. Molecular biology of the angiotensin I converting enzyme: I. Biochemistry and structure of the gene. J Hypertension 1993; 11: 471-476.

14. Costerousse O, Allegrini J, Huang H, Bounhik J, Alhenc-Gelas F. Regulation of ACE gene expression and plasma levels during rat postnatal development. Am J Physiol 1994; 267: E7454-E7453.

15. Weber KT, Brilla CG. Pathological hypertrophy and cardiac interstitium. Circulation 1991; 83: 1849-1865.

16. Brilla C, Weber K. Reactive and reparative myocardial fibrosis in arterial hypertensive in the rat. Cardiovasc Res 1992; 26: 671677.

17. Díez J, Laviades C, Varo N, Querejeta R, López B. Diagnóstico bioquímico de la fibrosis miocárdica hipertensiva. Rev Esp Car- diol 2000; 53 (Supl 1): 8-13.

18. Tan LB, Jalil JE, Pick R, Janicki JS, Weber KT. Cardiac myocyte necrosis induced by angiotensin II. Circ Res 1991; 69: 11851195.

19. Schunkert H, Dzau VJ, Tang SS, Hirsch AT, Apstein CS, Lorell $\mathrm{BH}$. Angiotensin II induced growth responses in isolated adult rat hearts. Evidence for load-independent induction of cardiac protein synthesis by angiotensin II. Circ Res 1995; 76: 489-497.

20. Katwa LC, Ratajska A, Cleutjens JPM, Sun Y, Zhou G, Lee SJ et al. Angiotensin converting enzyme and kininase-II-like activities in cultured valvular interstitial cells of the rat heart. Cardiovasc Res 1995; 29: 57-64.

21. Weber KT. Targeting pathological remodeling. Concepts of cardioprotection and reparation. Circulation 2000; 102: 1342-1345.

22. Challah M, Nicoletti A, Arnal JF, Philippe M, Laboulandine Y, Allegrini $\mathrm{J}$ et al. Cardiac angiotensin converting enzyme overproduction indicates interstitial activation in renovascular hypertension. Cardiovasc Res 1995; 30: 231-239.

23. Schunkert H, Jackson B, Tang SS, Schoen FJ, Smits JFM, Apstein CS et al. Distribution and functional significance of cardiac angiotensin converting enzyme in hypertrophied rat hearts. Circulation 1993; 87: 1328-1339.

24. Heymes C, Swynghedauw B, Chevalier B. Activation of angiotensinogen and angiotensin-converting enzyme gene expression in the left ventricle of senescent rats. Circulation 1994; 90: 13281333.

25. Falkenhahn M, Franke F, Bohle RM, Zhu YC, Stauss HM, Bachmann $\mathrm{S}$ et al. Cellular distribution of angiotensin-converting enzyme after myocardial infarction. Hypertension 1995; 25: 219226.

26. Hirsch AT, Talsness CE, Schunckert HS, Paul M, Dzau V. Tissue-specific activation of cardiac angiotensin converting enzyme in experimental heart failure. Circ Res 1991; 69: 475-482.

27. Sun Y, Cleutjens JPM, Díaz-Arias A, Weber KT. Cardiac angiotensin converting enzyme and myocardial fibrosis in the rat. Cardiovasc Res 1994; 28: 1423-1432.

28. Weber KT. Stroma and the search for common ground. Cardiovasc Res 1995; 29: 330-335.

29. Jalil JE, Doering CW, Janicki JS, Pick R, Shroff SG, Weber KT. Fibrillar collagen and myocardial stiffness in the intact hypertrophied left ventricle. Circ Res 1989; 64: 1041-1050.

30. Jalil JE, Janicki JS, Janicki JS, Shroff S, Pick R, Weber KT. Remodeling of coronary vasculature and myocardial fibrosis in renovascular hypertension: response to captopril. Am J Hypertens 1991; 4: 51-55.

31. Doering CW, Jalil JE, Janicki JS, Pick R, Aghili S, Abrahams C et al. Collagen network remodeling and diastolic stiffness of the rat left ventricle with pressure overload hypertrophy. Cardiovasc Res 1988; 22: 686-695.

32. Jalil JE, Doering CW, Janicki JS, Pick R, Clarck WA, Weber KT. Structural versus contractile protein remodeling and myocardial stiffness in the hypertrophied rat left ventricle. J Mol Cell Cardiol 1988; 20: 1179-1187.

33. Conrad CH, Brooks W, Hayes J, Sen S, Robinson K, Bing O. Myocardial fibrosis and stiffness with hypertrophy and heart failure in the spontaneously hypertensive rat. Circulation 1995; 91 : 161-170.

34. Schaison FH, Fernando Ramírez-Gil J, Ciferri S, Bernard M, Baudin B, Mougenot $\mathrm{N}$ et al. Acute and long-term dose-response study of quinapril on hormonal profile and tissue angiotensinconverting enzyme in Wistar rats. J Cardiovasc Pharmacol 1996; 28: 11-18.

35. Mukawa H, Toki Y, Shimauchi A, Matsui H, Morishima I, Okumura $\mathrm{K}$ et al. Pressure overload per se rather than cardiac angiotensin converting enzyme activity may be important in the development of rat cardiac hypertrophy. J Hypertens 1997; 15: 1027-1032.

36. Díez J, Panizo A, Hernández M, Vega F, Sola I, Fortuno MA et al. Cardiomyocyte apoptosis and cardiac angiotensin-converting enzyme in spontaneously hypertensive rats. Hypertension 1997; 
30: 1029-1034.

37. Montgomery HE, Kiernan LA, Whitworth CE, Fleming S, Unger $\mathrm{T}$, Gohlke $\mathrm{P}$ et al. Inhibition of tissue angiotensin converting enzyme activity prevents malignant hypertension in TGR (mREN2) 27. J Hypertens 1998; 16: 635-643.

38. Yoshida K, Kohzuki M, Casley DJ, Johnston CI. Angiotensinconverting enzyme inhibition and salt in experimental myocardial infarction. J Cardiovasc Pharmacol 1998; 32: 357-365.

39. Schieffer B, Wirger A, Meybrunn M, Seitz S, Holtz J, Riede UN et al. Comparative effects of chronic angiotensin-converting enzyme inhibition and angiotensin II type 1 receptor blockade on cardiac remodeling after myocardial infarction in the rat. Circulation 1994; 89: 2273-2282.

40. Duncan AM, Burrell LM, Kladis A, Campbell DJ. Effects of angiotensin-converting enzyme inhibition on angiotensin and bradykinin peptides in rats with myocardial infarction. J Cardiovasc Pharmacol 1996; 28: 746-754.

41. Ruzicka M, Skarda V, Leenen FH. Effects of ACE inhibitors on circulating versus cardiac angiotensin II in volume overload-induced cardiac hypertrophy in rats. Circulation 1995; 92: 3568-
3573.

42. Brilla CG, Pick R, Tan LB, Janicki JS, Weber KT. Remodeling of the rat right and left ventricles in experimental hypertension. Circ Res 1990; 67: 1355-1364.

43. Urata H, Healy B, Stewart RW, Bunpus FM, Husain A. Angiotensin II-forming pathways in normal and failing human hearts. Circ Res 1990; 66: 883-890.

44. Kinoshita A, Urata H, Bumpus FM, Husain A. Measurement of angiotensin I converting enzyme inhibition in the heart. Circ Res 1993; 73: 51-60.

45. Huang H, Arnal JF, Llorens-Cortes C, Challah M, Alhenc-Gelas $\mathrm{F}$, Corvol $\mathrm{P}$ et al. Discrepancy between plasma and lung angiotensin-converting enzyme activity in experimental congestive heart failure. A novel aspect of endothelium dysfunction. Circ Res 1994; 75: 454-460.

46. Linz W, Gohlke P, Unger T, Sholkens BA. Experimental evidence for effects of ramipril on cardiac and vascular hypertrophy beyond blood pressure reduction. Arch Mal Coeur 1995; 88: 31-34.

47. O'Sullivan JB, Black MJ, Bertram JF, Bobik A. Cardiovascular hypertrophy in one kidney, one clip hypertensive rats: a role for angiotensin II. J Hypertens 1994, 12: 1163-1170. 